\title{
BMJ Open The STOP-AB trial protocol: efficacy and safety of discontinuing patient antibiotic treatment when physicians no longer consider it necessary
}

\author{
Carl Llor, ${ }^{1}$ Ana Moragas, ${ }^{2}$ Carolina Bayona, ${ }^{3}$ Josep M Cots, ${ }^{4}$ José M Molero, ${ }^{5}$ \\ Joana Ribas, ${ }^{6}$ Julio Francisco Fóthy, ${ }^{7}$ Isabel Gutiérrez, ${ }^{8}$ Coro Sánchez, ${ }^{9}$ \\ Jesús Ortega, ${ }^{10}$ Javier Arranz, ${ }^{11}$ Jenifer Botanes, ${ }^{12}$ Purificación Robles ${ }^{13}$
}

To cite: Llor C, Moragas A, Bayona C, et al. The STOP-AB trial protocol: efficacy and safety of discontinuing patient antibiotic treatment when physicians no longer consider it necessary. BMJ Open 2017;0:e015814. doi:10.1136/ bmjopen-2016-015814

- Prepublication history and additional material is available. To view these files, please visit the journal online (http://dx.doi. org/10.1136/bmjopen-2016015814)

Received 2 January 2017 Revised 3 April 2017 Accepted 11 April 2017

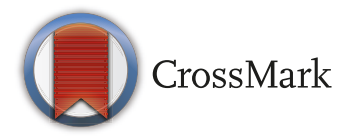

For numbered affiliations see end of article.

Correspondence to

Dr Carl Llor:

carles.llor@gmail.com

\section{ABSTRACT}

Introduction Since 2011, the Spanish Society of Family Medicine has recommended general practitioners (GPs) to ask their patients to stop taking antibiotics when they suspect a viral infection. However, this practice is seldom used because uncertainty about diagnosis, and fear of consequences of discontinuing antibiotic therapy, as well as perceived pressure to continue prescribing antibiotics and potential conflict with patients are more of a concern for GPs than antibiotic resistance. The main objective of this study is to determine whether discontinuation of antibiotic therapy when a GP no longer considers it necessary has any impact on the number of days with severe symptoms.

Methods and analysis This is a multicentre, openlabel, randomised controlled clinical trial. The study was conducted in 10 primary care centres in Spain. We included patients from 18 to 75 years of age with uncomplicated acute respiratory tract infectionsacute rhinosinusitis, acute sore throat, influenza or acute bronchitis-who had previously taken any dose of antibiotic for $<3$ days, which physicians no longer considered necessary. The patients were randomly assigned to the usual strategy of continuing antibiotic treatment or to discontinuing antibiotic therapy. A sample size of 240 patients per group was calculated on the basis of a reduction of 1 day in the duration of severe symptoms being a clinically relevant outcome. The primary outcome was the duration of severe symptoms, that is, symptoms scored 5 or 6 by means of validated symptom diaries. Secondary outcomes included antibiotics taken, adverse events, patient satisfaction and complications within the first 3 months.

Ethics and dissemination The study was approved by the Ethical Board of Fundació Jordi Gol i Gurina (reference number: 16/093). The findings of this trial will bedisseminated through research conferences and peerreviewed journals.

Trial registration number NCT02900820; pre-results.

\section{BACKGROUND}

Acute respiratory tract infections (RTIs) are among the most common reasons for a healthcare encounter in Western countries,
Strengths and limitations of this study

- The open design of this study will allow us to study the perceptions of patients in a situation similar to that of usual practice. Apart from its pragmatic design, our study will be the first trial to assess if discontinuation of antibiotic therapy when a general practitioner no longer considers it necessary has any impact on the number of days with severe symptoms.

- Some patients might not complete the symptom diaries. However, strategies to improve diary return rates will be implemented and reminder telephone calls will be made to non-respondents.

- The open nature of the study may cause a placebo effect favouring antibiotics. However, this effect will be minimised by the similar structured information all patients will receive about the self-limiting nature of respiratory tract infections and advice about the use of non-antibiotic medications.

- This trial might be underpowered for the detection of differences between the two groups in terms of adverse events and complications within the first 3 months, since these outcomes are considered secondary end points in this study.

- Another possible limitation of this study is the fact that microbiological studies will not be taken into account. In primary care, the response to treatment in respiratory tract infections is mainly judged by clinical rather than microbiological criteria.

accounting for about $15 \%$ of all visits. Most episodes are caused by viruses, and in otherwise healthy adults these infections are typically self-limiting and do not require a visit to a physician or a prescription medication. Nevertheless, many patients with uncomplicated, self-limited RTIs seek care in primary care offices. A recent study found that $72 \%$ of primary care visits due to an acute respiratory tract infection did not seem to require an office visit, ${ }^{2}$ which subsequently increases 
healthcare costs and often leads to inappropriate antibiotic prescription. ${ }^{3}{ }^{4}$ Very importantly, inappropriate overuse of antibiotic medications can be very detrimental and may lead to antibiotic resistance, ${ }^{56}$ which has a tremendous impact on the economy, ${ }^{7}$ and patients often experience adverse effects such as diarrhoea, thrush, nausea, urticaria and rash, which give rise to further office visits and time off work. Despite being uncommon, patients might also experience serious complications such as anaphylaxis and Clostridium difficile infection. ${ }^{8}$ In addition, medicalisation of a self-limited condition makes it more likely for patients to visit a healthcare provider the next time they have a similar episode. ${ }^{9}$

General practitioners (GPs) have generally been told to continue an antibiotic regimen once the patient has initiated it in order to prevent the patient from acquiring resistant organisms. However, this dogma of completing an antibiotic regimen once initiated might not be associated with less antimicrobial resistance. Some studies have shown that short-course regimens can be as effective as longer courses of therapy, resulting in less emergence of antibiotic resistance, which is consistent with what we know about natural selection, the driver of antibiotic resistance. ${ }^{10-12}$ The use of shorter therapies, defined as the taking of an antibiotic for 5 days or less, is commonly used for uncomplicated urinary tract infections. Short course regimens-from 3 to 5 days-have also shown to be as effective as longer therapies in community-acquired pneumonia, acute bacterial sinusitis and acute exacerbations of chronic obstructive pulmonary disease but are seldom used by GPs. ${ }^{13}$ When it comes to pneumonia, despite the efforts of infectious committees and guidelines developed by different societies, the duration of antibiotic use is still a major issue for which there is a lack of adherence both in primary and secondary care worldwide. $^{14}$

This often-heard statement of completing an antibiotic course once initiated and the perceived need to treat beyond resolution of symptoms is usually driven by a desire to prevent worse outcomes and relapses. Giving the right antibiotic at an adequate dose, along with good compliance with the daily regimen by the patient (ie, taking the correct dose at the appropriate intervals), has been said to be the most important strategy for treatment success in bacterial infections. ${ }^{15}$ However, GPs very often see patients with suspected viral infections of the upper and lower airways, for which antibiotic treatment makes little or no difference in terms of clinical outcomes and, conversely, can cause some side effects and might also prompt the acquisition of resistant organisms. ${ }^{16}$

Since 2011, the Spanish Society of Family Medicine has recommended GPs to ask their patients to stop taking antibiotics given by other clinicians when they suspect a viral infection. ${ }^{17}$ Despite this recommendation, this strategy is seldom used in routine clinical practice. GPs are reluctant and feel that it is unsafe to discontinue an antibiotic once the patient has already started it, mainly due to the ambiguity about the appropriateness of discontinuing medication felt by GPs, and in part because the clinical guidelines do not encourage discontinuation of medication, as they offer GPs a weak rationale for discontinuation. ${ }^{18}$ In addition, some studies have also shown that other issues such as uncertainty about diagnosis, ease of follow-up and fear of consequences of non-prescribing as well as perceived pressure to prescribe and potential conflict with patients which might lead to consequences for the future doctor-patient relationship are more of a concern for GPs continuing to prescribe antibiotics than antibiotic resistance. ${ }^{19}$ When it comes to acute infections, GPs might feel uncomfortable to discontinue antibiotic therapy from a patient who subsequently deteriorates, especially if the patient needs to be admitted to hospital. ${ }^{20}$ There is limited evidence about the efficacy and safety of continuing an antibiotic regimen if not needed, but concomitantly, evidence as to whether discontinuing antibiotic therapy for these conditions is safe is lacking, and studies demonstrating the safety of this practice should be carried out.

Discontinuing an antibiotic regimen could be undertaken in one of the following two situations:

1. Patients diagnosed with infectious diseases for which antibiotics-prescribed by other physicians-are not necessary, that is, an antibiotic course for suspected viral infections

According to WHO, $80 \%$ of the RTIs in the community have a viral origin. Most of these infections are self-limiting, and recent systematic reviews have suggested that antibiotics only slightly modify the course of most of these infections. A recent systematic review suggested that antibiotics do not improve the duration of symptoms in patients with common cold compared with those receiving placebo. ${ }^{21}$ Antibiotics are associated with modest benefits in sore throat; they reduce soreness and fever, the duration of symptoms and the incidence of suppurative and non-suppurative complications compared with placebo mainly in patients with infection caused by group A $\beta$-haemolytic streptococcus. ${ }^{22}$ This modest effect of antibiotics has also been observed in acute rhinosinusitis in which antibiotics can shorten the time to cure, but only five participants per 100 cure faster at any time point between 1 and 2 weeks if they receive antibiotics instead of placebo. ${ }^{23}$ In acute bronchitis, no difference has been shown between antibiotic and placebo groups in terms of the percentage of patients described as achieving clinical improvement at follow-up. ${ }^{24}$ However, the participants in all these clinical trials had a significantly greater risk of adverse effects with antibiotics than with placebo. ${ }^{25}$

Despite the low percentage of bacterial infections, antibiotic prescription is very high in these infectious diseases, with over $60 \%$ of adults presenting with acute rhinosinusitis, acute bronchitis and acute sore throat receiving an antibiotic in Spain. ${ }^{26}$ Antibiotic prescription in our country has increased over the last years and Spain now constitutes one of the leading countries in the world when it comes to the percentage of antibiotics prescribed. ${ }^{27} 28$ This unnecessary antibiotic prescription in RTIs has also 
been seen in other affluent countries; in addition, these antibiotics are increasingly broad-spectrum antimicrobial agents. ${ }^{29}{ }^{30}$ Even in Holland, the country with the lowest antibiotic consumption in Europe, $46 \%$ of the antibiotics prescribed for RTIs are not needed while in only $4 \%$ of the situations in which antibiotics are not prescribed they should have been recommended according to the Dutch guidelines. ${ }^{31}$ This unnecessary antibiotic prescription might mainly be explained by uncertainty in the diagnosis and by GPs' perceptions regarding patient expectations for a prescription. ${ }^{3233}$ Data from the Genomics to combat resistance against antibiotics in community-acquired lower respiratory tract infections in Europe study clearly show that antibiotics are expected by $45 \%$ of the patients with lower RTIs. ${ }^{34}$

2. Patients who have taken several doses of an antibiotic not prescribed by a health professional for an infectious disease for which antibiotics are not necessary, that is, from leftovers found in the household or an antibiotic bought without prescription at the pharmacy.

The sale of antibiotics and other antimicrobial medicines without a prescription remains widespread, with many countries lacking standard treatment guidelines, thereby increasing the potential for overuse of antimicrobial medicines by the public and medical professionals. ${ }^{35}$ This practice is common outside Northern Europe and North America. The percentage of non-prescription access to antimicrobials is often underestimated and also depends on the methodology used for making estimations. In 2016, the European Commission published a questionnaire-based study (Eurobarometer), which was carried out in 28 European countries, including 1053 respondents in Spain. This study described that $6 \%$ of users reported having obtained antibiotics in the previous year without a prescription or stated that they had used the leftovers from a previous course ${ }^{36}$ However, when more reliable methods are used to know how many individuals obtain antibiotics from sources other than their GPs, these percentages clearly increase ${ }^{37}$ Self-medication with antimicrobials is also widespread, occurring among the population in the same countries in which over-thecounter sales are available. ${ }^{38}$

\section{STUDY AIMS}

The main objective of the present trial is to know if the discontinuation of antibiotic therapy when a GP no longer considers it necessary has any impact on the number of days with severe symptoms.

Secondary objectives:

1. Assessment of the incidence of adverse effects.

2. Assessment of antibiotic consumption.

3. Assessment of the satisfaction with healthcare and belief in the effectiveness of antibiotics by the patients included.

4. Assessment of the number of complications observed within the first 3 months.

\section{METHODS AND ANALYSIS}

\section{Study design}

This was a multicentre, open-label, randomised controlled clinical trial comparing two therapeutic strategies for uncomplicated acute RTIs. Patients were randomly assigned to either the usual strategy of continuing antibiotic treatment or discontinuing antibiotic therapy. The randomisation was stratified by condition of interestacute sore throat, rhinosinusitis (including common cold), acute bronchitis and influenza. GPs randomised patients using a centralised electronic online platform. An openlabel study has been designed taking into account the type of the two strategies used and considering that an effect on GPs' and patients' beliefs is expected. Neither patients nor health professionals will be blinded.

\section{Eligibility criteria}

Any patient from 18 to 75 years of age attending the GP consultation with an uncomplicated RTI who had previously taken any dose of antibiotic for $<3$ days due to either of the two following clinical scenarios and accepted to participate in the clinical trial were included:

- Patients diagnosed with clinical conditions for which antibiotics prescribed by other health professionals are not necessary.

- Patients who have taken several doses of an antibiotic (from leftovers found in the household or obtained at the pharmacy without a medical prescription) for a clinical condition for which an antibiotic is not necessary.

\section{Exclusion criteria}

- Subjects under 18 or over 75 years of age.

- Confirmed bacterial infection.

- Patients requiring hospital admission.

- Severe impairment of clinical signs of infection (impairment of consciousness, respiratory rate $>30$ respirations per minute, heart rate $>125$ beats per minute, systolic blood pressure $<90 \mathrm{~mm} \mathrm{Hg}$, diastolic blood pressure $<60 \mathrm{~mm} \mathrm{Hg}$, temperature $>40^{\circ} \mathrm{C}$, oxygen saturation $<92 \%$ ).

- Problems to comply with treatment at home, such as sociopathy or psychiatric problems or drug or alcohol addiction.

- Lack of tolerance to oral treatment, such as the presence of nausea and vomiting, gastrectomy, postsurgery and/or diarrhoea.

- Significant comorbidity including severe renal failure, hepatic cirrhosis, severe heart failure, immunosuppression-chronic HIV infection, transplantation, neutropaenia or patients receiving immunosuppressive drugs or corticosteroids.

- Terminal disease, defined as a life expectancy $<12$ months.

- Patients admitted to a long-term residence.

- Patients who state that they are unable to see their doctor at the practice office.

- Refusal to participate in the study. 
Table 1 Symptom diary. For each symptom choose the degree of affectation in the last 24 hours, according to the following Table of Codes.

\begin{tabular}{ll}
\hline Code & Explanation \\
\hline 0 & Normal/does not hurt \\
1 & Affectation is insignificant/mild pain \\
2 & Mild or slightly significant affectation/moderate pain \\
3 & Moderately significant affectation/considerable pain \\
4 & Significant affectation/intense pain \\
5 & Highly significant affectation/very intense pain \\
\hline 6 & Maximum affectation/unbearable pain \\
\hline
\end{tabular}

Acute sore throat. The following symptoms were recorded daily: feeling of fever, headache, general discomfort or malaise, cough, sore throat, difficulty swallowing (solids or liquids), runny nose, ear pain, diarrhoea, nausea, vomiting, difficulty sleeping and difficulty in carrying out daily life activities. Each symptom was scored following a $0-6$ scale.

Rhinosinusitis. The following symptoms were recorded daily: feeling of fever, general discomfort or malaise, headache, sudden pain in the face, pain in the face on touching, runny nose, nasal mucus colour, cough, sore throat, expectoration or phlegm (mucus when coughing), diarrhoea, nausea, vomiting, difficulty sleeping and difficulty in carrying out daily life activities. Each symptom (save for nasal mucus colour) was scored from 0 to 6 . In the case of the presence of nasal mucus colour, the colour was assessed: transparent $(T)$, light yellow clear $(\mathrm{Y})$, green $(\mathrm{V})$ or other $(\mathrm{O})$. Acute bronchitis. The following symptoms were recorded daily: feeling of fever, general discomfort or malaise, cough, expectoration or phlegm (mucus when coughing), shortness of breath (suffocation, fatigue), pain when breathing (chest pain), chest breathing sounds, diarrhoea, nausea, vomiting, difficulty sleeping and difficulty in carrying out daily life activities. Each symptom (save for expectoration or phlegm) was scored from 0 to 6 . In the case of expectoration or phlegm, the colour was assessed: transparent $(\mathrm{T})$, light yellow clear $(\mathrm{Y})$, green $(\mathrm{V})$ or other (O).

Influenza. The following symptoms were recorded daily: feeling of fever, runny nose, headache, general discomfort or malaise, cough, muscle pain, sore throat, sweat or chills, shortness of breath, weakness or fatigue, dizziness, diarrhoea, nausea or vomiting, difficulty sleeping and difficulty in carrying out daily life activities. Each symptom was scored from 0 to 6 .

\section{Outcome measures}

The primary outcome measure was the duration of severe symptoms. Each symptom was scored using a six-point Likert scale and symptoms scoring 5 or 6 was considered as severe. We included common symptoms such as feeling of fever, discomfort or general malaise, cough, difficulty in sleeping and changes in everyday life in all patients, and specific symptoms according to the condition (table 1). ${ }^{39}$

Secondary end points

- Adverse events in the two study groups.

- Antibiotic consumption. This information was collected in the symptom diaries and also by the pharmacy units of the different healthcare systems.

- Patient satisfaction with healthcare by means of a questionnaire with a Likert scale.

- Patient belief in the effectiveness of antibiotics by means of a questionnaire with a Likert scale.
- Complications related to the RTIs were registered during the first 3 months after randomisation. As in the recent study by Gulliford $e t a l$, we considered the cases of pneumonia, empyema, peritonsillar abscess, mastoiditis, otitis media, bacterial meningitis and intracranial abscess. ${ }^{40}$ These complications were prospectively recorded by the GPs by means of a standardised questionnaire and were reported within a maximum of 48 hours to the study clinical coordinator, who reported to the Safety and Data Monitoring Committee, which assessed the safety of the two strategies.

\section{Sample size calculation}

Excellence guidelines for the treatment of RTIs included estimates of average duration of the illness (before and after seeing a doctor) of 1 week for acute sore throat, one and a half weeks for rhinosinusitis, including common cold and 3 weeks for acute cough or bronchitis. ${ }^{39}$ In a recent study on the effectiveness of delayed prescribing strategies carried out in Spain, the mean duration of severe symptoms in uncomplicated acute RTIs including acute sore throat, rhinosinusitis and acute bronchitis, among patients not treated with antibiotics was 4.7 days (SD of 3.6).$^{39}$ Considering a reduction of 1 day in the duration of severe symptoms as a clinically relevant outcome, with a bilateral approximation, a sample of 240 patients per group was able to detect this difference with an alpha error of $5 \%$ and a power of $80 \%$ (beta $=0.2$ ), considering $15 \%$ of losses (based on the percentage of patients who did not return symptom diaries in a previous study).$^{39}$ In terms of the number of investigators to be included in this clinical trial, we anticipated that over a 2-year period each GP would include 32 patients. Thus, we are planning to invite 15 GPs from 10 different healthcare centres.

\section{Data collection and ascertainment of visits}

The patients were randomised to one of the two treatment strategies. Baseline data were collected in the clinic by the physician or with the help of the nursing staff. To standardise data collection, all of the participating healthcare professionals were trained by the coordinating centre. Only experienced GPs (those who have worked for 15-25 years) and those who feel comfortable with the design of the study participated in the study. This was achieved by the administration of a questionnaire with clinical questions, recommendations of local guidelines and vignettes to check if they were confident and comfortable with the strategy of stopping an already commenced antibiotic course. The patients received information on the study and, if they were interested in participating, they provided with an informed consent form to read and sign. A maximum length of 15-20 min was expected for the interview and the introduction of the data. The study scheme and the visit programme were explained to the patient (table 2). GPs filled out a screening log with all the patients who met all the inclusion criteria and none of the exclusion criteria regardless of whether the patients 


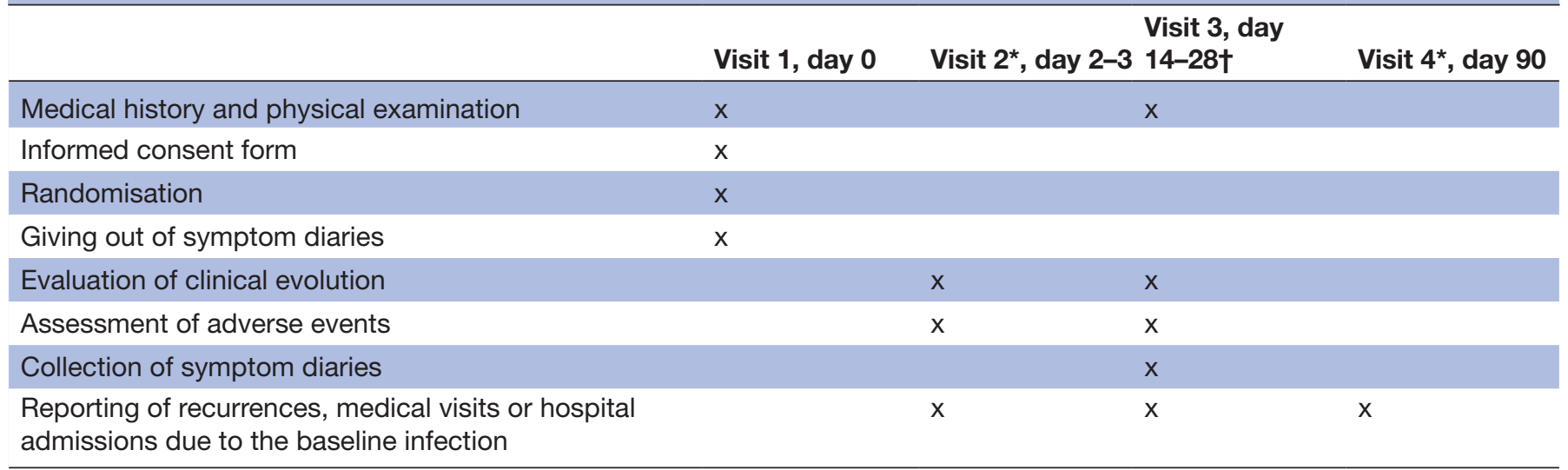

*Phone call.

†Depending on the infection (14 days for influenza and sore throat; 28 days for acute bronchitis and acute rhinosinusitis). A further visit should be scheduled if symptoms persist on visit 3.

consent to participate or not. This allowed us to evaluate the percentage of patients who accepted to participate in the trial and determined the reasons why they did not wish to participate if they refuse.

On the baseline visit, GPs collected information about the type of diagnoses, prior time elapsed with symptoms, number of days taking an antibiotic and type of antibiotic taken. Patients who made the decision to take an antibiotic by themselves (either purchased at the pharmacy or taken from leftovers stored at home) and were assigned to the usual strategy of continuing antibiotic treatment were provided with a medical prescription of the same antibiotic until completing the recommended therapy duration according to local guidelines, even if the antibiotic was not first-line treatment.

After randomisation, information on the strategy to which they have been allocated was given to the participants, and they were informed as to the appropriate measures to take in case their condition worsened or there was no improvement. In addition, they were given a diary with a validated questionnaire of symptoms for each condition, which they had to complete while symptoms related to the respiratory condition were present. The degree of satisfaction or concern with different aspects of the therapy as well as the use of antibiotics was also recorded in this diary.

Patients were interviewed by telephone 2 or 3 days after their inclusion in the study. At this first follow-up visit, a worsening of the clinical situation of the patient was evaluated to determine whether antibiotic treatment was necessary among patients in the group assigned to discontinuation (first-line antibiotics were recommended in this case) or whether the antibiotic regimen should be continued or changed to the first-line drug in patients in the group allocated to continuation. We provided the patients with the opportunity to ask questions and discuss any problems with diary completion. In addition, compliance and possible secondary effects of the treatment were evaluated. The second follow-up visit was scheduled at 14-28 days after inclusion and depended on the infection involved at patient recruitment as established in the National Institute for Health and Care Excellence guidelines. ${ }^{41}$ On this visit, the clinical evolution of the signs and symptoms were evaluated and the need to change or continue the antibiotic treatment, respectively, was determined in the case of worsening of the clinical manifestations. The symptom diaries were collected on this visit. Participants who did not return their diary were telephoned by the trial team as a reminder and to enquire whether they needed any assistance in returning their diary. Possible secondary effects of the treatment were also assessed. Patients were asked to revisit if symptoms continued or they presented recurrence of the infection. On the last follow-up visit, scheduled at day 90, recurrences, medical visits or hospital admissions due to the baseline infectious disease were reported.

\section{Data analysis procedure}

The characteristics of the study population will be described using frequencies for categorical variables and mean and SD for quantitative variables. A bivariate analysis of the baseline data will be performed between patients returning and not returning symptoms diaries for assessing if the latter population differ from the patients included in the study analysis. To compare the two strategies studied, we will use the t test for continuous variables. To compare the duration of symptoms across strategies, we will use the test for continuous variables for each symptom, and a linear regression model per symptom, with symptom duration as the dependent variable, and both antibiotic consumption and symptom duration prior to the visit as the independent variables. The same approach will be made to compare the severity of symptoms between the two strategies, but the dependent variable for each model will be symptom severity. The $\chi^{2}$ test will be used to compare antibiotic consumption, satisfaction, percentage of adverse effects and the 
appearance of complications between the two groups. The level of significance will be $5 \%(\alpha=0.05)$.

Criteria for withdrawals were evidence of protocol violation, withdrawal of informed consent or a serious adverse event, defined as any untoward medical circumstance that results in death, is life-threatening, requires inpatient hospitalisation or prolongs existing hospitalisation, results in persistent or significant disability/incapacity or requires intervention to prevent permanent impairment or damage. Patients may choose to interrupt the medication at any time during the course of the study. However, they were followed in the same way as the other patients. Analyses will follow the intention-to-treat principle.

\section{ETHICS AND DISSEMINATION}

Each patient provided written consent to participate in the study after being informed in intelligible language for him/her on the nature, scope and possible consequences of the trial. After consent was submitted, the patients were randomised. Data confidentiality was ensured at all times, as stated in the researcher's commitment sheet, as will compliance with the current legislation regarding the protection of personal data. This was a clinical trial based on the outpatient setting, and neither patients nor researchers received any monetary compensation. From an ethical point of view, this is to certify that the objective of the study was relevant for primary care, the power of the study may be considered as reasonable, this was an original study, the risks which the participants may incur justify the study being carried out with a totally favourable benefit/risk quotient and we ensured the external validity of the study to the primary care reality, with clearly described inclusion and exclusion criteria. Vulnerable populations did not participate in this study.

The trial has been registered with the National Institutes of Health (NIH) trial registry (NCT02900820). This protocol was presented in the last Conference of the Spanish Society of Family Medicine held in La Coruña in June 2016. A range of dissemination activities are planned at national and international conferences. We will publish the final report in an open access peer-review journal (see online supplementary material). A summary of the findings will be sent to the participating practices on completion of the STOP Antibiotic Regimen (STOPAB) study.

\section{DISCUSSION}

Overprescribing of antibiotics for RTIs is considerable in Western countries. The results of this study will be applicable to patients diagnosed with clinical conditions for which antibiotics are not or might not be necessary. Few GPs ask patients to discontinue antibiotic therapy because of fear of possible worsening of symptoms and the appearance of complications even in cases of suspected viral infections. Guidelines usually provide dominating triggers for prescribing and weak priming for discontinuation. In other situations, GPs do not wish to contradict the decision made by other doctors because it might look unfair. If the results of this study do not find any difference between the two groups, there will be no reason to continue an antibiotic regimen when a GP no longer considers it necessary. We believe that there is no riskand every advantage-in stopping a course of an antibiotic immediately after a bacterial infection has been excluded or is unlikely, as Gilbert recently suggested. ${ }^{16}$ The most obvious circumstances in which it is appropriate to stop antibiotics are when the antibiotics are initiated without certainty of what infection is being treated, if any treatable bacterial infection is present at all, and for infections that are almost always self-limiting, for example, episodes of acute bronchitis. Patient expectation often plays a role in the decision to start antibiotic treatment in these cases.

The impact of our trial is likely to be important since the evidence available in this field is still limited, and adequately designed and conducted clinical trials are warranted to clarify the efficacy and safety of discontinuing antibiotic therapy in these cases. Similarly, the inclusion of most of the uncomplicated RTIs and the fact that only patients with $<3$ days of antibiotic therapy will be invited to participate will increase the validity of the results. These characteristics make this study innovative and relevant to this field.

\section{Author affiliations}

${ }^{1}$ Primary Healthcare Centre Barcelona-2B (Via Roma), Barcelona, Spain

${ }^{2}$ Universitat Rovira i Virgili, Primary Healthcare Centre Jaume I, Tarragona, Spain

${ }^{3}$ Primary Healthcare Centre La Marina, Barcelona, Spain

${ }^{4}$ Universitat of Barcelona, Primary Healthcare Centre La Marina, Barcelona, Spain

${ }^{5}$ Primary Healthcare Centre San Andrés, Madrid, Spain

${ }^{6}$ Primary Healthcare Centre Hospital Son Llàtzer, Palma, Spain

${ }^{7}$ Primary Healthcare Centre Coll d'en Rabassa, Palma, Spain

${ }^{8}$ Primary Healthcare Centre Rondilla I, Valladolid, Spain

${ }^{9}$ Primary Care Centre Virxe da Peregrina, Pontevedra, Spain

${ }^{10}$ Primary Healthcare Centre Rincón de Soto, Rincón de Soto, Spain

${ }^{11}$ Primary Healthcare Centre Arquitecte Bennàssar, Palma, Spain

${ }^{12}$ Primary Healthcare Centre Cornellà de Llobregat, Barcelona, Spain

${ }^{13}$ Primary Care Centre Can Vidalet, Esplugues de Llobregat, Spain

Contributors $\mathrm{CL}, \mathrm{AM}, \mathrm{JMC}$ and $\mathrm{CB}$ were involved in the conception and design of the study. JFF, JMM, JR, IG, JA, JO, CS, JB and PR have made substantial contributions to conception and design. AM, JFF, JMM, JR, IG, JA, J0, CS, JB and PR were involved in trial conduct and recruitment. CL drafted the manuscript and all authors read and approved the final manuscript.

Funding This work is supported by the Catalan Society of Family Medicine, grant number FAP1601. Neither the funding society nor the sponsor - Spanish Society of Family and Community Medicine - had any role in the design of the study.

Competing interests $\mathrm{CL}$ reports receiving research grants from the European Commission (Sixth and Seventh Programme Frameworks), Catalan Society of Family Medicine and Instituto de Salud Carlos III (Spanish Ministry of Health).

Ethics approval The study was approved by the Primary Care Research and Ethics Committee of the Jordi Gol i Gurina Foundation, Barcelona, Spain (reference number, 16/093).

Provenance and peer review Not commissioned; externally peer reviewed.

Data sharing statement All data created during this research will be openly available from the Spanish Society of Family Medicine.

Open Access This is an Open Access article distributed in accordance with the Creative Commons Attribution Non Commercial (CC BY-NC 4.0) license, which permits others to distribute, remix, adapt, build upon this work non-commercially, and license their derivative works on different terms, provided the original work is 
properly cited and the use is non-commercial. See: http://creativecommons.org/ licenses/by-nc/4.0/

(C) Article author(s) (or their employer(s) unless otherwise stated in the text of the article) 2017. All rights reserved. No commercial use is permitted unless otherwise expressly granted.

\section{REFERENCES}

1. Hak E, Rovers MM, Kuyvenhoven MM, et al. Incidence of GPdiagnosed respiratory tract infections according to age, gender and high-risk co-morbidity: the second Dutch National Survey of General Practice. Fam Pract 2006;23:291-4

2. Renati S, Linder JA. Necessity of office visits for acute respiratory infections in primary care. Fam Pract 2016;33:312-7.

3. Linder JA, Stafford RS. Antibiotic treatment of adults with sore throat by community primary care physicians: a national survey, 1989-1999. JAMA 2001;286:1181-6.

4. Grijalva CG, Nuorti JP, Griffin MR. Antibiotic prescription rates for acute respiratory tract infections in US ambulatory settings. JAMA 2009;302:758-66.

5. Goossens H, Ferech M, Vander Stichele R, et al. Outpatient antibiotic use in Europe and association with resistance: a cross-national database study. Lancet 2005;365:579-87.

6. Costelloe C, Metcalfe C, Lovering A, et al. Effect of antibiotic prescribing in primary care on antimicrobial resistance in individual patients: systematic review and meta-analysis. BMJ 20102096;340:c2096;340:c2096.

7. Smith $\mathrm{R}$, Coast $\mathrm{J}$. The true cost of antimicrobial resistance. BMJ 2013;346:f1493.

8. Armbruster S, Goldkind L. A 5-year retrospective review of experience with Clostridium difficile-associated diarrhea. Mil Med 2012;177:456-9.

9. Little P, Gould C, Williamson I, et al. Reattendance and complications in a randomised trial of prescribing strategies for sore throat: the medicalising effect of prescribing antibiotics. BMJ 1997;315:350-2.

10. Singh N, Rogers P, Atwood CW, et al. Short-course empiric antibiotic therapy for patients with pulmonary infiltrates in the intensive care unit. A proposed solution for indiscriminate antibiotic prescription. Am J Respir Crit Care Med 2000;162(2 Pt 1):505-11.

11. Bell BG, Schellevis F, Stobberingh $E$, et al. A systematic review and meta-analysis of the effects of antibiotic consumption on antibiotic resistance. BMC Infect Dis 2014;14:13.

12. Schrag SJ, Peña $C$, Fernández $J$, et al. Effect of short-course, highdose Amoxicillin therapy on resistant pneumococcal carriage: a randomized trial. JAMA 2001;286:49-56.

13. Spellberg B. The New Antibiotic Mantra-"Shorter Is Better". JAMA Intern Med 2016;176:1254-5.

14. Viasus D, Vecino-Moreno M, De La Hoz JM, et al. Antibiotic stewardship in community-acquired pneumonia. Expert Rev Anti Infect Ther 2017;15:351-9.

15. Ball P, Baquero F, Cars O, et al. Antibiotic therapy of community respiratory tract infections: strategies for optimal outcomes and minimized resistance emergence. J Antimicrob Chemother 2002;49:31-40.

16. Gilbert GL. Knowing when to stop antibiotic therapy. Med J Aust 2015;202:121-2.

17. Grupo de Enfermedades Infecciosas. Sociedad Española de Medicina Familiar Y Comunitaria. 10 mandamientos - Uso antibióticos. Decálogo para un uso más racional de los antibióticos para profesionales sanitarios. $2011 \mathrm{https} / /$ www.semfyc.es/es/ grupos_trabajo/infecciosas/noticias_grupo/detalle/dia_mundial_ resistencias_2011/ (accessed 2 Apr 2017).

18. Nixon MS, Vendelø MT. General practitioners' decisions about discontinuation of medication: an explorative study. J Health Organ Manag 2016;30:565-80.
19. Tonkin-Crine S, Yardley L, Little P. Antibiotic prescribing for acute respiratory tract infections in primary care: a systematic review and meta-ethnography. J Antimicrob Chemother 2011;66:2215-23.

20. Kumar S, Little P, Britten N. Why do general practitioners prescribe antibiotics for sore throat? grounded theory interview study. BMJ 2003;326:138.

21. Kenealy T, Arroll B. Antibiotics for the common cold and acute purulent rhinitis. Cochrane Database Syst Rev 2013;6:CD000247.

22. Spinks A, Glasziou PP, Del Mar CB. Antibiotics for sore throat. Cochrane Database Syst Rev 2013;(11):CD000023.

23. Lemiengre MB, van Driel ML, Merenstein D, et al. Antibiotics for clinically diagnosed acute rhinosinusitis in adults. Cochrane Database Syst Rev 2012;10:CD006089.

24. Smith SM, Fahey T, Smucny J, et al. Antibiotics for acute bronchitis. Cochrane Database Syst Rev 2014;(3):CD000245.

25. Arroll B. Antibiotics for upper respiratory tract infections: an overview of Cochrane reviews. Respir Med 2005;99:255-61.

26. Llor C, Cots JM, Hernández S, et al. Effectiveness of two types of intervention on antibiotic prescribing in respiratory tract infections in primary care in Spain. happy Audit Study. Aten Primaria 2014:46:492-500.

27. Versporten A, Bolokhovets G, Ghazaryan L, et al. Antibiotic use in Eastern Europe: a cross-national database study in coordination with the WHO Regional Office for Europe. Lancet Infect Dis 2014;14:381-7.

28. Laxminarayan $\mathrm{R}$, Sridhar $\mathrm{D}$, Blaser $\mathrm{M}$, et al. Achieving global targets for antimicrobial resistance. Science 2016;353:874-5.

29. Barnett ML, Linder JA. Antibiotic prescribing to adults with sore throat in the United States, 1997-2010. JAMA Intern Med 2014;174:138-40.

30. Barnett ML, Linder JA. Antibiotic prescribing for adults with acute bronchitis in the United States, 1996-2010. JAMA 2014;311:2020-2.

31. Dekker AR, Verheij TJ, van der Velden AW. Inappropriate antibiotic prescription for respiratory tract indications: most prominent in adult patients. Fam Pract 2015;32:401-7.

32. Whaley LE, Businger AC, Dempsey PP, et al. Visit complexity, diagnostic uncertainty, and antibiotic prescribing for acute cough in primary care: a retrospective study. BMC Fam Pract 2013;14:120

33. Cockburn J, Pit S. Prescribing behaviour in clinical practice: patients expectations and doctors' perceptions of patients' expectations--a questionnaire study. BMJ 1997;315:520-3.

34. Coenen S, Francis N, Kelly M, et al. Are patient views about antibiotics related to clinician perceptions, management and outcome? A multi-country study in outpatients with acute cough. PLoS One 2013;8:e76691.

35. Morgan DJ, Okeke IN, Laxminarayan R, et al. Non-prescription antimicrobial use worldwide: a systematic review. Lancet Infect Dis 2011;11:692-701.

36. Special Eurobarometer 445, report. Antimicrobial resistance, June 2016. ISBN 978-92-79-58818-1. http://ec.europa.eu/dgs/health_ food-safety/amr/docs/eb445_amr_generalreport_en.pdf. (accessed 2 Apr 2017).

37. Llor C, Moragas A. Pros and cons of the over-the-counter sales of antimicrobials. WMJ 2015;61:64-8.

38. Grigoryan L, Haaijer-Ruskamp FM, Burgerhof JG, et al. Selfmedication with antimicrobial drugs in Europe. Emerg Infect Dis 2006;12:452-9.

39. de la Poza Abad M, Mas Dalmau G, Moreno Bakedano M, et al. Delayed antibiotic Prescription (DAP) Group. prescription strategies in acute uncomplicated respiratory infections: a randomized clinical trial. JAMA Intern Med 2016;176:21-9.

40. Gulliford MC, Moore MV, Little P, et al. Safety of reduced antibiotic prescribing for self limiting respiratory tract infections in primary care: cohort study using electronic health records. BMJ 2016;354:i3410.

41. NICE short clinical guidelines Technical Team. Rrespiratory tract infections-antibiotic prescribing. Prescribing of antibiotics for selflimiting respiratory tract infections in adults and children in primary care. London, UK: National Institute for Health and Care Excellence, 2008. 